\title{
Editorial
}

\section{Plant-Derived Antioxidants in Disease Prevention}

\author{
Renata Szymanska, ${ }^{1}$ Pavel Pospisil, ${ }^{2}$ and Jerzy Kruk ${ }^{3}$ \\ ${ }^{1}$ Department of Medical Physics and Biophysics, Faculty of Physics and Applied Computer Science, \\ AGH University of Science and Technology, Reymonta 19, 30-059 Krakow, Poland \\ ${ }^{2}$ Department of Biophysics, Faculty of Science, Centre of the Region Haná for Biotechnological and Agricultural Research, \\ Šlechtitelů 27, Palacký University, 78371 Olomouc, Czech Republic \\ ${ }^{3}$ Department of Plant Physiology and Biochemistry, Faculty of Biochemistry, Biophysics and Biotechnology, \\ Gronostajowa 7, 30-387 Krakow, Poland \\ Correspondence should be addressed to Renata Szymanska; renata.szymanska@fis.agh.edu.pl
}

Received 1 December 2016; Accepted 1 December 2016

Copyright @ 2016 Renata Szymanska et al. This is an open access article distributed under the Creative Commons Attribution License, which permits unrestricted use, distribution, and reproduction in any medium, provided the original work is properly cited.

Plant-derived antioxidants are a large group of natural products with reducing or radical-scavenging capacity. Due to their potent preventive, as well as therapeutic actions, these compounds receive a great deal of attention by not only scientists but also pharmacologists and physicians.

Maintenance of redox homeostasis plays a central role in health and disease prevention. Oxidative stress is generated by unbalance between reactive oxygen species (ROS) and antioxidants. Excess of ROS leads to degradation of lipids, proteins and nucleic acids and thus may lead to oxidative damage of cells and in a consequence to overexpression of oncogenes, mutagens formation, induction of atherogenic activity, or inflammation. Oxidative stress is suggested to play a major role in pathogenesis of cardiovascular diseases, neurodegeneration, cancers, immune disorders, diabetes, aging, and others. Plants, especially dietary fruits and vegetables, are a rich source of antioxidants. It is postulated that antioxidants show health benefits through direct reduction of oxidative stress. In the body, antioxidant network works in concert through several different mechanisms: ROS scavenging, termination of lipid peroxidation, or chelating of metals. Despite the fact that broad knowledge of antioxidant structures, properties, and biological actions has been gathered, many aspects required clarification and further studies. Relatively little is known about cellular mechanisms of their therapeutic potential, interactions with other compounds, appropriate dosage, and effectiveness of a treatment (especially their effect in randomized clinical trials). Furthermore, the bioactiveness of a large number of natural compounds remains unknown.
Well-known antioxidants, as well as those newly discovered, raise hopes for their use in the prevention and treatment of the above-mentioned diseases.

In this special issue, an attempt has been made to gather articles that update our understanding about the role of plantderived antioxidants in disease prevention. These reports filled the gaps in the field of antioxidants research, allow better understanding of their action, and facilitate their future usage in diseases prevention and treatment. The special issue complies sixteen (16) excellent articles including four (4) reviews, eleven (11) research papers, and one (1) clinical study, which show current and recent developments in plantderived antioxidant research.

The review by S. Perrone et al. summarizes the latest knowledge about the role of plant-derived xanthophyll, lutein, as an antioxidant and anti-inflammatory agent in animal model and human, with special focus on newborns. An interesting review by Y. Zhao et al. describes mechanism of action and the clinical status of ligustrazine (tetramethylpyrazine), a natural compound from Ligusticum wallichii, which is extensively used in Chinese medicine for treatment of such diseases as cardiovascular disorders, diabetes, cancers, or liver injury. S. Ojha et al.s review is focused on the role on plant-derived compounds for counteracting cisplatin-induced nephrotoxicity. Cisplatin is a commonly used chemotherapeutic, which very often induces organ toxicity, in particular of kidneys and ears. Thus, the authors describe the most suitable plant-originated compounds that are able to combat cisplatin-induced nephrotoxicity. A nice 
"methodological" review provided by A. M. Pisoschi et al. gathers the information about analytical methods which can be applied for measurements of antioxidants and antioxidant capacity of plant-derived products. This article describes the antioxidant extraction procedures and discusses advantages and disadvantages of commonly used analytical methods for antioxidant detection.

ROS play a major role in pathogenesis of numerous diseases. It is believed that application of exogenous antioxidants is a promising strategy to suppress oxidative stress associated with those disorders or via other still not recognized mechanisms. In this special issue most of research articles show that plant-derived compounds (or their extracts) acts successfully as suppressors of oxidation and can be used in the future as therapeutic agents. The published papers show data obtained either on (1) cell lines or (2) on the animal models. The research article by P.-H. Li et al. shows that ethyl acetate and dichloromethane extracts of medicinal plant Equisetum ramosissimum inhibit the growth of human malignant melanomas. Authors in in vitro experiments tried also to explain the mechanism of their action, which probably is connected with caspase induction. Likewise, J. Ferreira Campos et al. evaluated the chemical profile of ethanol extract of Senna velutina leaves and showed its cytotoxic effect against leukemic cells. Next, Plinia peruviana (Jaboticaba) fruit peels, which is a rich source of phenolic compounds and well-known antioxidant, were examined by H. S. Pitz et al's group. They have reported that a hydroalcoholic extract of this Brazilian plant has a positive potential during the wound healing. In turn, B. Zhou et al. have explored the effect of soy oligopeptides in UVB-induced acute photodamage of human skin. Topically applied soy oligopeptides showed protective effects by increased $\mathrm{Bcl}-2$ protein expression and decreased CDPs-positive cells, sunburn cells, apoptotic cells, p53, and Bax proteins expression in epidermis layer of UVB-irradiated foreskin.

R. H. Oliveira Lopes et al. conducted the research on animal models. They treated hyperlipidemic Wistar rats with hydroethanolic extract of Curatella americana leaves. They have demonstrated that, as compared to the control, this extract contains compounds that have ability to lower cholesterol and triglycerides levels in tested rats. The study of $\mathrm{M}$. Xu et al. showed prophylactic effect of resveratrol on the incidence of portal vein system thrombosis in rat fibrosis model. The protective effects of resveratrol, among others, were connected with decrease in platelet aggregation, ROS production, and increase of nitric oxide synthesis. P. P. de Toledo Espinodola et al. group have also evaluated the antihyperlipidemic effects of plant extract. They tested root water extract of guavira (Campomanesia adamantium) on lipid profile of hyperlipidemic Wistar rats. The extract, rich in phenolic compounds (mostly gallic and ellagic acids), had ability to lower lipid peroxidation and improve lipid serum level in animals. R. M. Perez-Gutierrez et al. isolated six novel flavonoids from the bark of Eysenhardtia polystachya. They tested their activity on streptozotocin-induced diabetic mice and found that newly discovered phenolics are potent antioxidants and can have therapeutic effects in diabetes. M. Kluknavsky et al. examined the effect of (-)-epicatechin on locomotor activity and hypertension in commonly used animal model of hypertension, spontaneously hypertensive rats. (-)-Epicatechin treatment prevented from hypertension and reduced locomotor hyperactivity which was, among others, the results of increase of nitric oxide synthase and total antioxidants capacity in the blood. H. Li et al. studied the protective effect of astragalan, an acidic polysaccharide isolated from medicinal plant, Astragalus membranaceus, against neurotoxin 6-hydroxydopamine that can cause parkinsonism. Experiments, which were performed on Caenorhabditis elegans models, have shown that Astragalus polysaccharide shows a great therapeutic potential in neurodegeneration treatment. R. C. L. Affonso et al. investigated whether aqueous extract of coffee bean residual press cake improved the skin wound healing. They found that coffee extracts significantly reduced wound area size on the inflammatory phase.

The article provided by X. Li et al. describes the results of clinical trial combined with preclinical studies on rat model. A clinical trial experiments confirmed the association of oxidants/antioxidants unbalance with coronary chronic total occlusion. They examined 399 patients at age of 8089. The preclinical studies encompass rat treatment with a polysaccharide isolated from endothelium corneum gigeriae galli. In both cases the authors have measured the level of superoxide dismutase 3, nitric oxide, endothelial nitric oxide synthase, and malondialdehyde. The obtained results have shown that the tested polysaccharide could be used as a potential therapeutic agent for coronary chronic total occlusion in very old patients.

Taken altogether, the data presented in this special issue cover a series of topics addressing the role of plant-derived antioxidants in different oxidative stress-related diseases prevention. We believed that the papers published in this special issue enrich our understanding of a physiological action of natural products but also provide promising perspectives on their future usage as therapeutic agents. We are sure that all the information provided in this issue will be of broad interest.

\section{Acknowledgments}

We would like to thank all the authors, reviewers, and editorial staff who contributed to the preparation of this special issue.

Renata Szymanska

Pavel Pospisil

Jerzy Kruk 


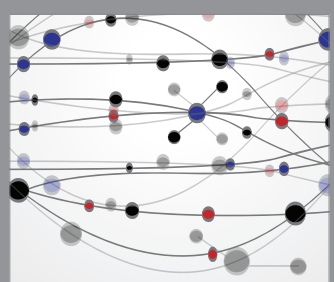

The Scientific World Journal
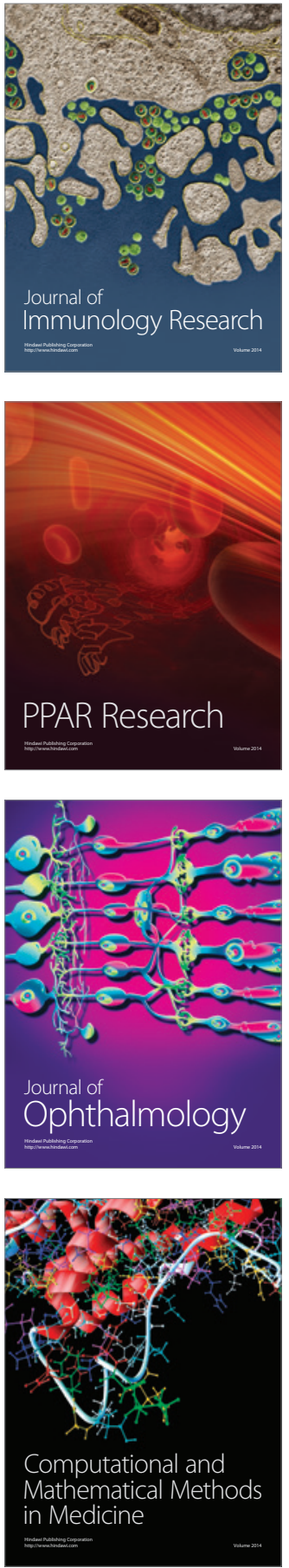

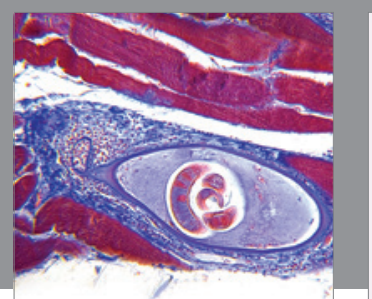

Gastroenterology Research and Practice

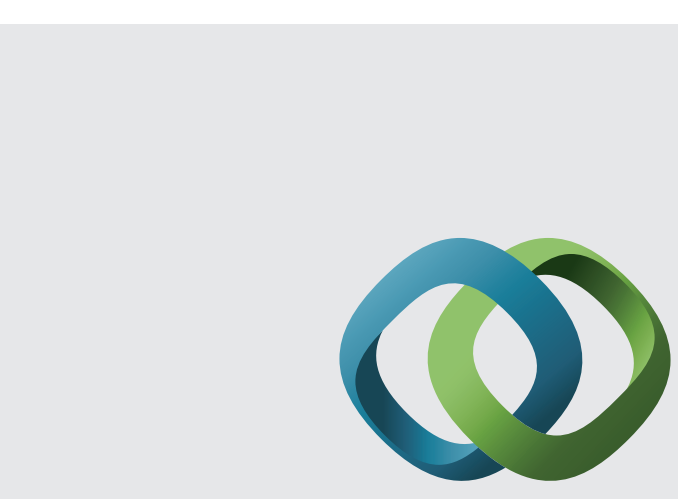

\section{Hindawi}

Submit your manuscripts at

http://www.hindawi.com
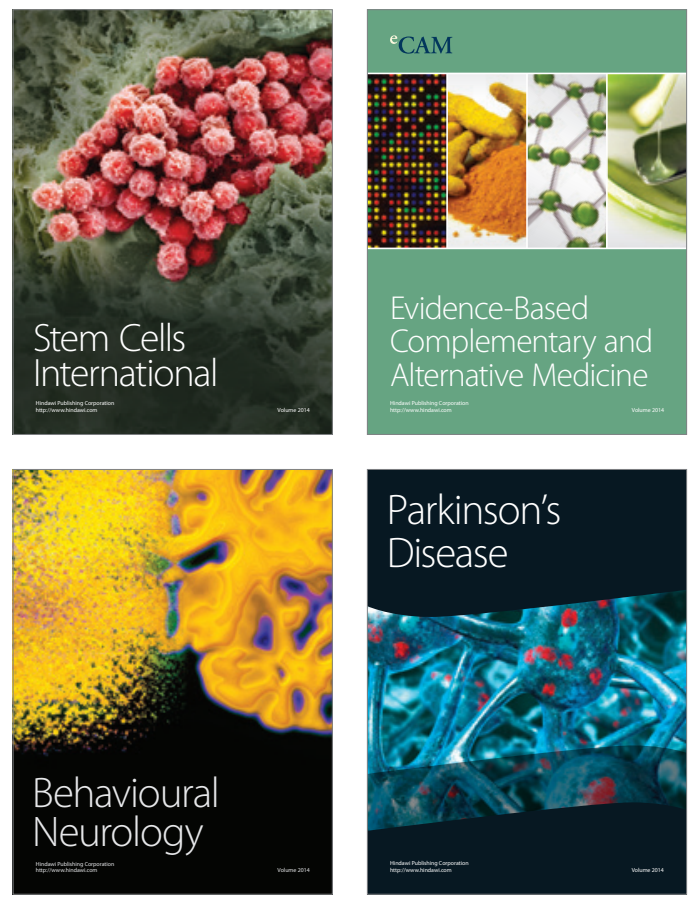
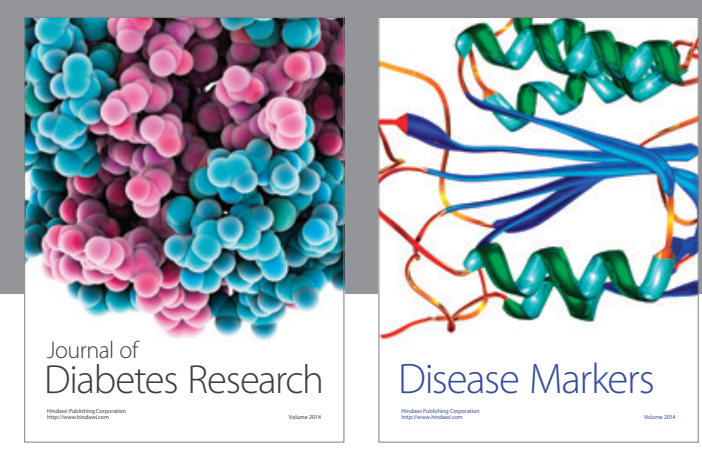

Disease Markers
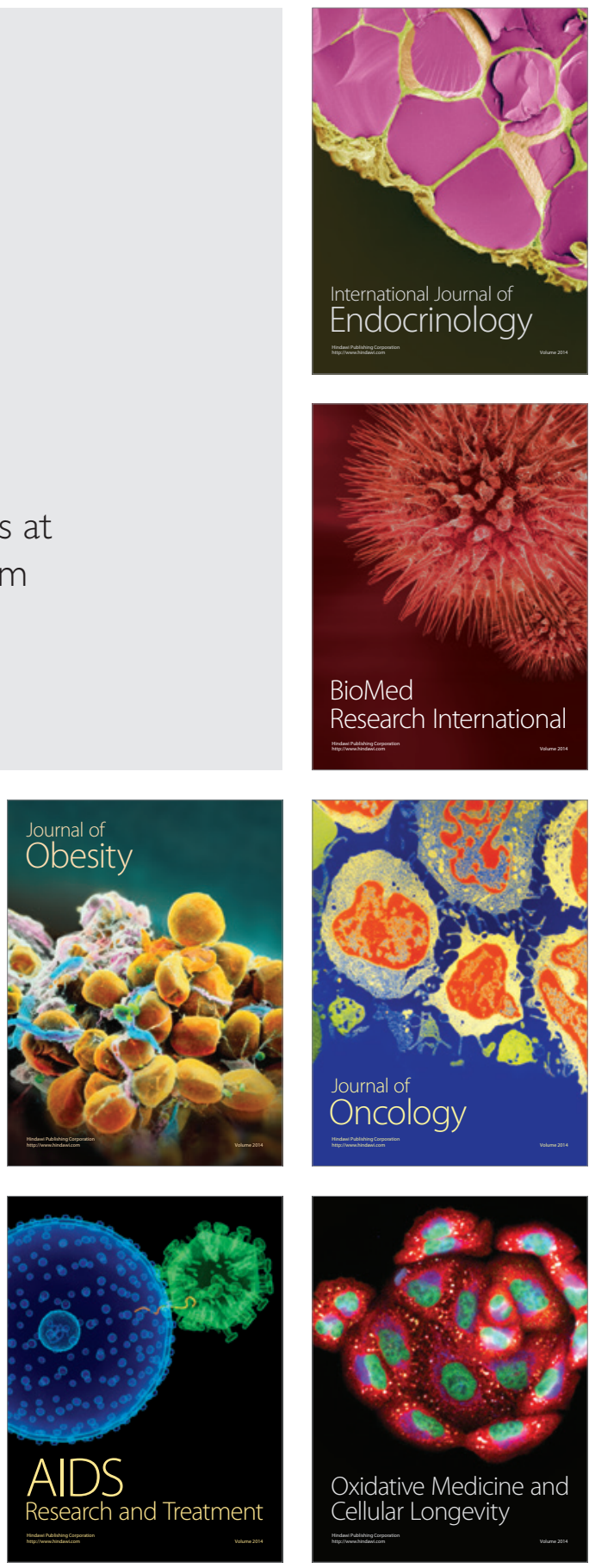